\title{
Performance Enhancement of Routing Protocol for Longer Life Time of WSN
}

\author{
Neha Choudhary \\ M-Tech Research Scholar \\ Department of Computer Science and \\ Engineering, NRI Institute of Information \\ Science and Technology, Bhopal
}

\author{
Vaibhava Singh \\ Research Guide \\ Department of Computer Science and \\ Engineering, NRI Institute of Information \\ Science and Technology, Bhopal
}

\begin{abstract}
Wireless sensor networks are the key part of mobile ad-hoc network (MANET) and the every node has to be active for long time to send information on the server. Since it has been operated with battery so there is a challenge to keep such node active for long time. The way nodes are active for long period of time is to change the pattern of data transfer between node and the server. To achieve this goal several routing protocols came under existence. One of them is low energy adaptive clustering hierarchy $(\mathrm{LEACH})$ is performing better lifetime of the WSN. In this paper the LEACH routing protocols is modified and made more energy efficient by changing the selection procedure of the cluster heads. By making the probability little bit higher for cluster head selection makes lifetime of the cluster longer than existing work.
\end{abstract}

\section{Keywords}

WSN, MANET, Cluster Head and LEACH.

\section{INTRODUCTION}

A wireless sensor network is a collection of sensor nodes with limited power supply and constrained computational and transmission capability. Due to the limited transmission and computational ability, and high density of sensor nodes, forwarding of data packets takes place in multi-hop data transmission. As a result routing in wireless sensor networks has been an important area of research in the past few years. The sensor nodes run on non-rechargeable batteries, so along with efficient routing the network should be energy efficient with efficient utilization of the resources and hence this is an important research concern. Advances in wireless evolution of low cost sensor nodes have led to introduction of low power wireless sensor networks. Due to multiple functions and ease of deployment of the sensor nodes it can be used in various applications such as target tracking, environment monitoring, health care, forest fire detection, inventory control, surveillance, energy management, and reconnaissance, and so on [1]. The main responsibility of the sensor nodes in a network is to forward the collected information from the source to the sink for further operations, but the resource limitations [2], unreliable links between the sensor nodes in combination with the various application demands of different applications make it a difficult task to design an efficient routing algorithm in wireless sensor networks.

Designing suitable routing algorithms for different applications, fulfilling the different performance demands has been considered as an important issue in wireless sensor networks. In this context many routing algorithms have been proposed to improve the performance demands of various applications through the network layer of the wireless sensor networks protocol stack $[3,4]$, but most of them are based on single-path routing. In single-path routing approach basically source selects a single path which satisfies the performance demands of the application for transmitting the load towards the sink.

\section{ROUTING IN WSN}

Since transmission of data from the targeted source to the sink is the main task of the wireless sensor networks, the technique used to do the data forwarding is an important issue which should be considered in developing these networks. Considering the unique features of low power wireless sensor networks, routing in WSN is much more challenging compared to traditional wireless networks such as ad-hoc networks $[3,4]$. First, considering the high density of nodes, the routing protocols should route data over long distances, regardless of the network structure and size, in addition to the above requirement some of the active nodes may fail during the operations due to the environmental factors or energy depletion of sensor nodes or hardware faults, but that issues shall not interrupt the normal operations of the network. Moreover, as mentioned earlier the wireless sensor nodes are restricted in terms of power supply, processing capability and available bandwidth, routing and data forwarding should be performed with effective network resource utilization. Further, considering the performance demands of the wireless sensor networks are totally application dependent, routing algorithms should satisfy the QoS demands of the application for which the network is being deployed. For example, challenges in designing the routing algorithms for environment monitoring will be different from issues that should be considered for health care monitoring or target tracking.

A clustering based routing algorithm called Low Energy Adaptive Clustering Hierarchy (LEACH) was proposed as a solution for low power consumption. LEACH arranges the nodes in the network into small clusters and chooses one of them as the cluster-head. Node senses its target and then sends the relevant information to its cluster-head. Then the cluster head aggregate and compress the information received from all the nodes and sends it to the base station. The nodes chosen while the cluster head drain out more energy as compared to the other nodes as it is required to send data to the base station which may be far located. Therefore LEACH use random rotation of the nodes required to be the cluster-heads to evenly distribute energy consumption in the network. 


\section{PROPOSED APPROACH}

The energy aware wireless sensor network using LEACH protocol is better to save energy of the nodes and increases the life time of the sensor network. To work little bit better for the same network is and considering same routing protocol a methodology is proposed which significantly enhances the performance the lifetime of the wireless sensor network (WSN). The idea behind the enhancement of the lifetime of the network is simple changing the selection pattern of the cluster heads $(\mathrm{CHs})$ in $\mathrm{LEACH}$ routing protocol.

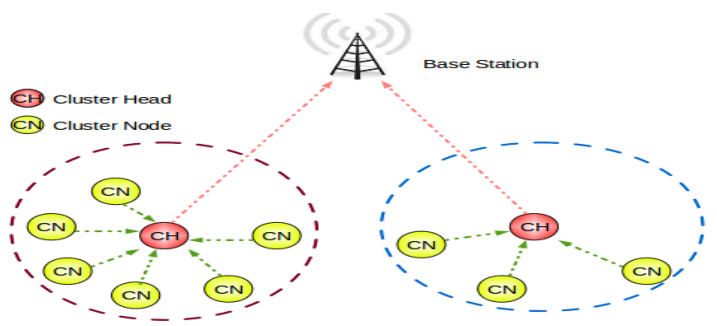

Fig.1 LEACH Network Routing Protocol

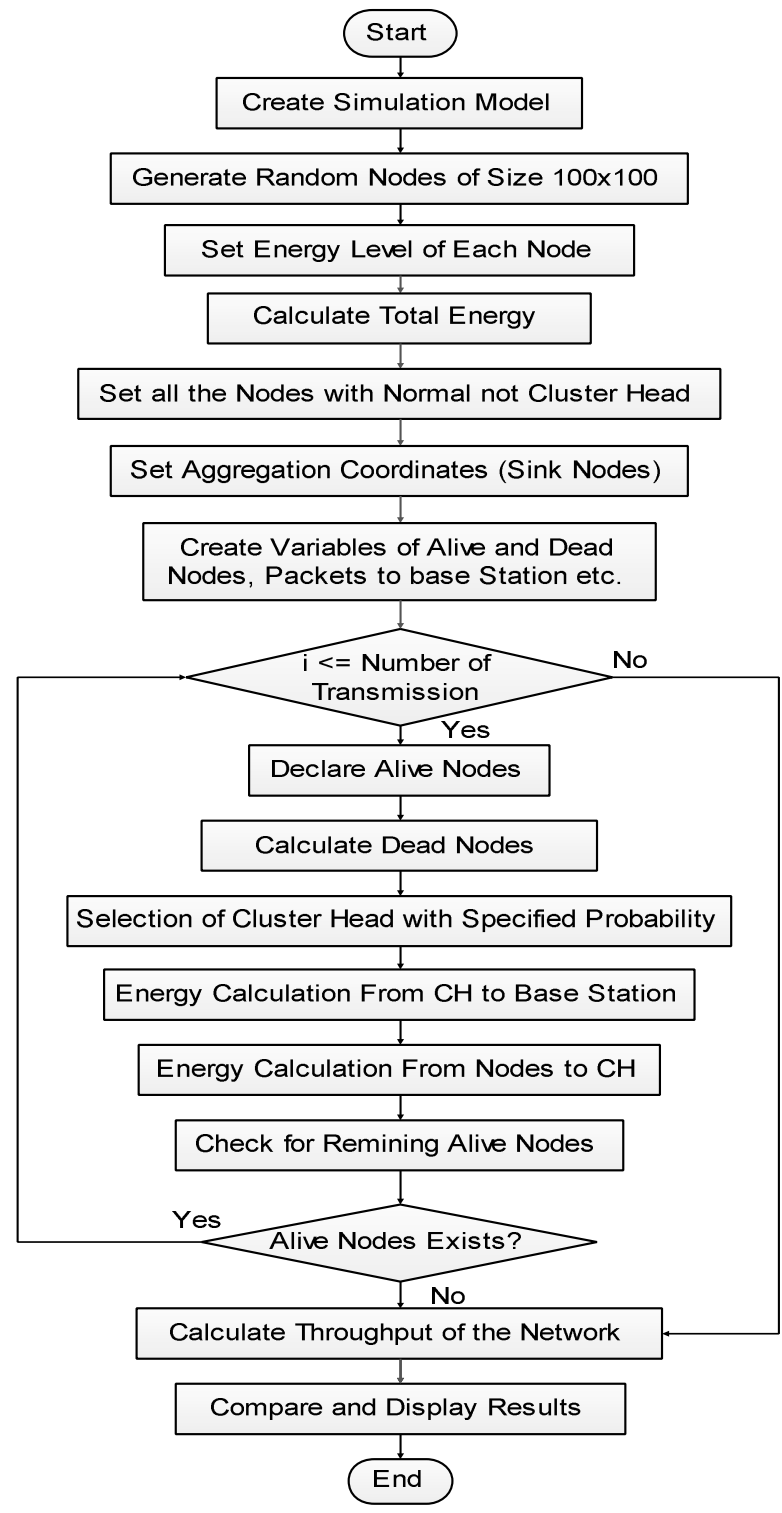

Fig. 2 Flow chart of the proposed LEACH based routing Protocol
The criteria of $\mathrm{CH}$ selection in the LEACH routing protocol is about $5 \%$ and eureka moment is if the probability of the $\mathrm{CH}$ selection is increased more than $5 \%$ than the lifetime of the network will definitely going to increase. The cluster diagram if the LEACH is shown in the figure below. The green circles are normal nodes and the red circles are cluster heads $(\mathrm{CHs})$ which was selected periodically after each transmission to the base station (BS).

The proposed LEACH protocol is implemented and the flow of the algorithm is given in the Fig. 2.2. The major steps are:

- $\quad$ Start of Simulation

- Create Simulation Model with the size of the network and set the alive nodes with the specific priority and energy

- Start of transmission with the assignment of cluster head and calculations of the different energy dissipation e.g. energy consumption from node to cluster head( $\mathrm{CH})$ and energy consumption from cluster head $(\mathrm{CH})$ to base station(BS)

- By performing several round of transmission calculate the number of alive nodes and energy remained

- Calculate dead nodes and the alive nodes

- Calculate of the throughput of the network

- Compare and display results

- end of simulation

\section{SIMULATION RESULTS}

The proposed model explained in this paper is implemented as the flow chart shown.

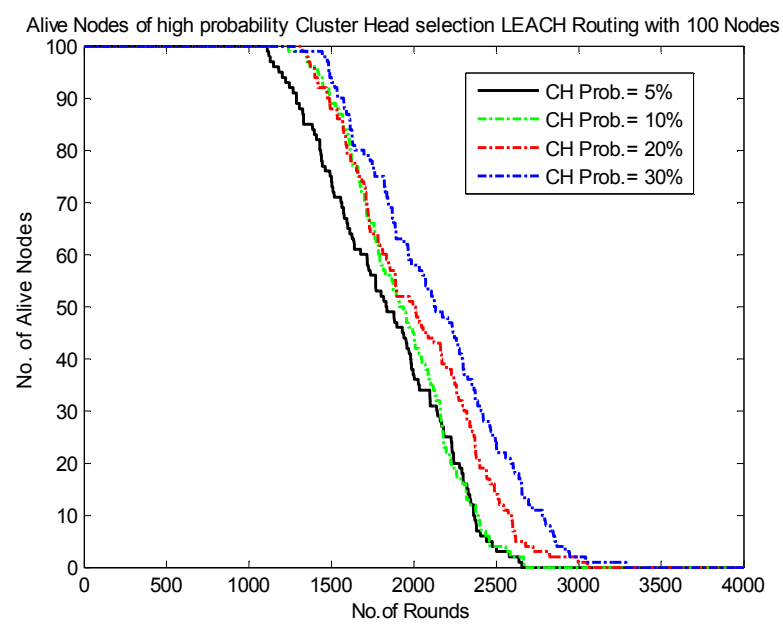

Fig. 3 Network lifetime in terms of dead nodes versus number of rounds

The simulated outcomes are in terms of number of alive nodes and number of dead nodes versus number of transmission rounds and throughput curve.

In the previous work lifetime of the network with low energy adaptive clustering hierarchy (LEACH) is calculated up to 2490 transmission rounds. If the network sustain for more number of rounds means lifetime of the network is going better. In proposed approach the lifetime of the network 
sustain up to 3341 rounds which greater than the previous work.

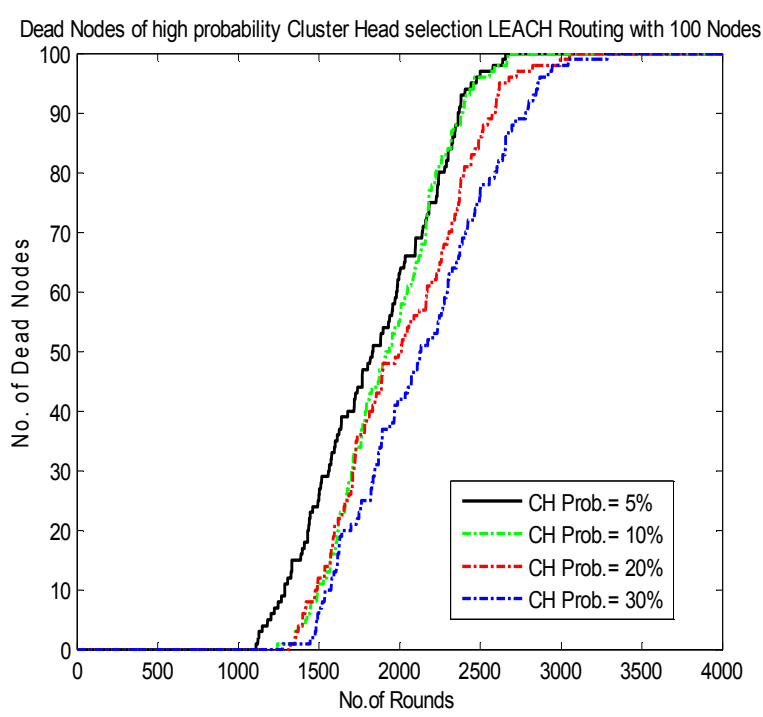

Fig. 4 Network lifetime in terms of alive nodes versus number of rounds

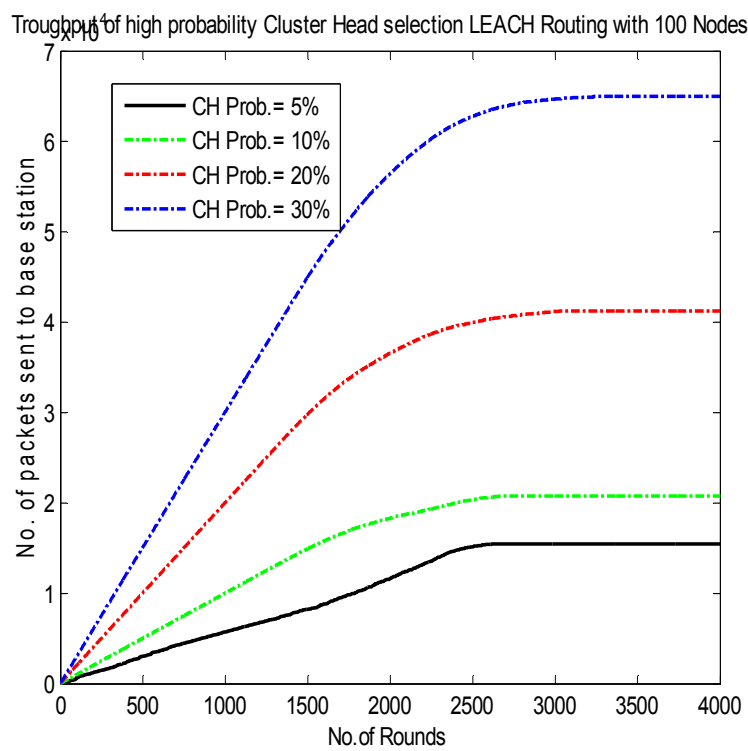

Fig. 5 Throughput versus no. of rounds

Table I: Radio Parameters

\begin{tabular}{|l|l|}
\hline Operation & Energy Dissipated \\
\hline $\begin{array}{l}\text { Transmitter } \\
\text { Electronics }\end{array}$ & Eelec $=$ Etx $=$ Erx $=50 \mathrm{~nJ} / \mathrm{bit}$ \\
\hline Data aggregation energy & EDA $=5 \mathrm{~nJ} / \mathrm{bit} /$ signal \\
\hline $\begin{array}{l}\text { Transmit amplifier } \\
\text { (if } \mathrm{d} \text { to BS }<\text { do) }\end{array}$ & Efs $=10 \mathrm{pJ} / \mathrm{bit} / 4 \mathrm{~m} 2$ \\
\hline $\begin{array}{l}\text { Transmit amplifier } \\
(\text { if } d \text { to BS }>\text { do) }\end{array}$ & Emp $=0.0013 \mathrm{pJ} / \mathrm{bit} / \mathrm{m} 4$ \\
\hline
\end{tabular}

Table II: Comparison of DDR, LEACH and LEACH-C for Scalability

\begin{tabular}{|l|l|l|l|l|}
\hline \multirow{4}{*}{$\begin{array}{l}\text { Routing } \\
\text { Protocol }\end{array}$} & Network Area & $\begin{array}{l}\text { Number } \\
\text { of Nodes }\end{array}$ & $\begin{array}{l}\text { First } \\
\text { Node } \\
\text { Death } \\
\text { Round }\end{array}$ & $\begin{array}{l}\text { Last } \\
\text { Node } \\
\text { Death } \\
\text { Round }\end{array}$ \\
\hline \multirow{4}{*}{$\begin{array}{l}\text { Proposed } \\
\text { Approach }\end{array}$} & $100 \mathrm{mX100m}$ & 100 & 1257 & 3341 \\
\cline { 2 - 5 } & $134 \mathrm{mX134m}$ & 134 & 1072 & 3073 \\
\cline { 2 - 5 } & $200 \mathrm{mX} 200 \mathrm{~m}$ & 200 & 918 & 3202 \\
\hline \multirow{4}{*}{ DDR } & $100 \mathrm{mX} 100 \mathrm{~m}$ & 100 & 1496 & 2490 \\
\cline { 2 - 5 } & $134 \mathrm{mX134m}$ & 134 & 1460 & 2459 \\
\cline { 2 - 5 } & $150 \mathrm{mX} 150 \mathrm{~m}$ & 150 & 1424 & 2388 \\
\cline { 2 - 5 } & $200 \mathrm{mX} 200 \mathrm{~m}$ & 200 & 1204 & 2270 \\
\hline
\end{tabular}

\section{CONCLUSION AND FUTURE SCOPE}

The energy efficient routing protocol analyzed and simulated in this paper has better approach to increase the lifetime of the wireless sensor network compared to existing work. As shown in the simulation results the alive nodes exists up to 3341 rounds of transmission and the throughput is greater than $6 \times 10-4$. With this proposed approach the network lasts longer than the routing techniques proposed before. If the routing is better advanced with the timing access considerations it will have longer network lifetime with the proposed work.

\section{REFERENCES}

[1] Jennifer Yick, Biswanath Mukherjee, and Dipak Ghosal. Wireless sensor network survey. Comput. Netw., 52(12):2292-2330, August 2008

[2] Kamalrulnizam Abu Bakar Marjan Radi, Behnam Dezfouli andMalrey Lee. Multipath routing in wireless sensor networks: Survey and research challenges. MDPI Sensors, 12(1):650-685, January 2012.

[3] J. N. Al-karaki and A. E. Kamal. Routing techniques in wireless sensor networks: A survey. IEEE Wireless Communications, 11(6):6-28, December 2004.

[4] Kemal Akkaya and Mohamed Younis. A survey on routing protocols for wireless sensor networks. Ad Hoc Networks, 3:325-349, 2005.

[5] Jamal N. Al-karaki and Ahmed E. Kamal. Routing techniques in wireless sensor networks: A survey. IEEE Wireless Communications, 11:6-28, 2004.

[6] Y. Geng C. Hong-bing and H. Su-jun. Nhrpa: a novel hierarchical routing protocol algorithm for wireless sensor networks. China Universities of Posts and Telecommunications, September 2008.

[7] G. Hu D. Wu and G. Ni. Research and improve on secure routing protocols in wireless sensor networks. In 4th IEEE International Conference on Circuits and Systems for Communications (ICCSC 2008). 
[8] Wendi Rabiner Heinzelman, Anantha $\mathrm{Ch}$, and Hari Balakrishnan. Energy-efficient communication protocol for wireless microsensor networks. pages 3005-3014, 2000.

[9] Dongjin Son, Bhaskar Krishnamachari, and John Heidemann. Experimental study of concurrent transmission in wireless sensor networks. In Proceedings of the 4th international conference on Embedded networked sensor systems, SenSys '06, pages 237-250, New York, NY, USA, 2006. ACM.

[10] Wenjing Lou,Wei Liu, and Yanchao Zhang. Performance optimization using multipath routing in mobile ad hoc and wireless sensor networks. In MaggieXiaoyan Cheng, Yingshu Li, and Ding-Zhu Du, editors, Combinatorial Optimization in Communication
Networks, volume 18 of Combinatorial Optimization, pages 117-146. Springer US, 2006.

[11] Chalermek Intanagonwiwat, Ramesh Govindan, and Deborah Estrin. Directed diffusion: a scalable and robust communication paradigm for sensor networks. In Proceedings of the 6th annual international conference on Mobile computing and networking, MobiCom '00, pages 56-67, New York, NY, USA, 2000. ACM.

[12] Wendi Rabiner Heinzelman, Joanna Kulik, and Hari Balakrishnan. Adaptive protocols for information dissemination in wireless sensor networks. In Proceedings of the 5th annual ACM/IEEE international conference on Mobile computing and networking, MobiCom '99, pages 174-185, New York, NY, USA, 1999. ACM. 